\title{
Calendar of forthcoming meetings
}

\section{Gang Wang}

Research \& Development Center for Functional Crystals, Institute of Physics, Chinese Academy of Sciences, No. 8 Nansanjie, Zhongguancun, Haidian District, Beijing 100190, China gangwang@iphy.ac.cn

\section{2-26 April 2019}

2019 MRS Spring Meeting \& Exhibit

Phoenix, Arizona, USA

[Info: https://www.mrs.org/spring2019]

5-10 May 2019

Gordon Research Conference on Neutron ScatteringEmerging Neutronic Approaches for Advanced Materials Study and Innovation in Energy, the Environment, Health and Infrastructure

Hong Kong, China

[Info: https://www.grc.org/neutron-scattering-conference/2019/]

9-12 May 2019

Pharmaceutical Powder X-ray Diffraction Symposium (PPXRD-16)

Paul Scherrer Institute, Switzerland

[Info: http://www.icdd.com/index.php/ppxrd/]

2-7 June 2019

8th International Conference on Hard X-ray Photoelectron Spectroscopy (HAXPES 2019)

Synchrotron SOLEIL, Paris, France

[Info: https://www.synchrotron-soleil.fr/en/events/haxpes-2019]

\section{3-28 June 2019}

11th International Conference on Inelastic X-ray Scattering (IXS2019)

Stony Brook University, New York, USA

[Info: https://www.bnl.gov/ixs2019/]

\section{3-28 June 2019}

Gordon Research Conference on Crystal Growth and Assembly-New Paradigms in the Mechanistic Understanding of Ordered Materials Assembly

University of New England, Biddeford, Maine, USA

[Info: https://www.grc.org/crystal-growth-and-assemblyconference/2019/]

1-5 July 2019

European Conference on Neutron Scattering 2019 (ECNS 2019)

Moskovskye Vorota Congress Center, Saint Petersburg, Russia

[Info: http://ecns2019.com/]
8-11 July 2019

8th International Meeting of the Union for Compact Accelerator-Driven Neutron Sources (UCANS-8)

Paris, France

[Info: https://ucans8.sciencesconf.org/]

20-24 July 2019

American Crystallographic Association Annual Meeting 2019

Covington, Kentucky, USA

[Info: https://www.amercrystalassn.org/2019-meeting]

28 July-2 August 2019

19th International Conference on Crystal Growth and Epitaxy (ICCGE-19)

Keystone, Colorado, USA

[Info: https://www.iccge19.org/]

28 July-2 August 2019

Gordon Research Conference on X-ray Science

Stonehill College, Easton, Massachusetts, USA

[Info: https://www.grc.org/x-ray-science-conference/2019/]

\section{5-9 August 2019}

Joint Meeting-68th Annual Denver X-ray Conference 2019 \& 25th International Congress on X-ray Optics and Microanalysis (ICXOM-25)

The Westin Lombard Yorktown Center, Lombard, Illinois, USA

[Info: http://www.dxcicdd.com/]

\section{8-23 August 2019}

32nd European Crystallographic Meeting

Vienna, Austria

[Info: https://ecm2019.org/home/]

1-4 September 2019

17th European conference on Solid State Chemistry (17th ECSSC)

Lille, France

[Info: https://ecssc17.com/]

8-11 September 2020

21st International Symposium on Industrial Crystallization

Potsdam, Germany

[Info: https://dechema.de/en/ISIC_21.html] 
22-25 September 2019

2019 GSA Annual Meeting

Phoenix, Arizona, USA

[Info: https://www.geosociety.org/GSA/Events/Annual_Meeting/ GSA/Events/gsa2019.aspx]

\section{3-26 September 2019}

3rd J-PARC Symposium (J-PARC2019)

Tsukuba, Japan

[Info: http://j-parc.jp/symposium/j-parc2019/]
19-22 November 2019

$\mathrm{X}$-rays and Matter

Domaine de l'Asnée, Villers-les-Nancy, France

[Info: http://www.rayonsxetmatiere.org/]

1-6 December 2019

2019 MRS Fall Meeting

Boston, Massachusetts, USA

[Info: https://www.mrs.org/fall2019]

****** (All information above as of 7 January 2019) ****** 